\title{
Intestinal radiopacities in chronic renal failure
}

\author{
Roland Schmitt MD, Hermann Haller MD, Joachim Lotz MD
}
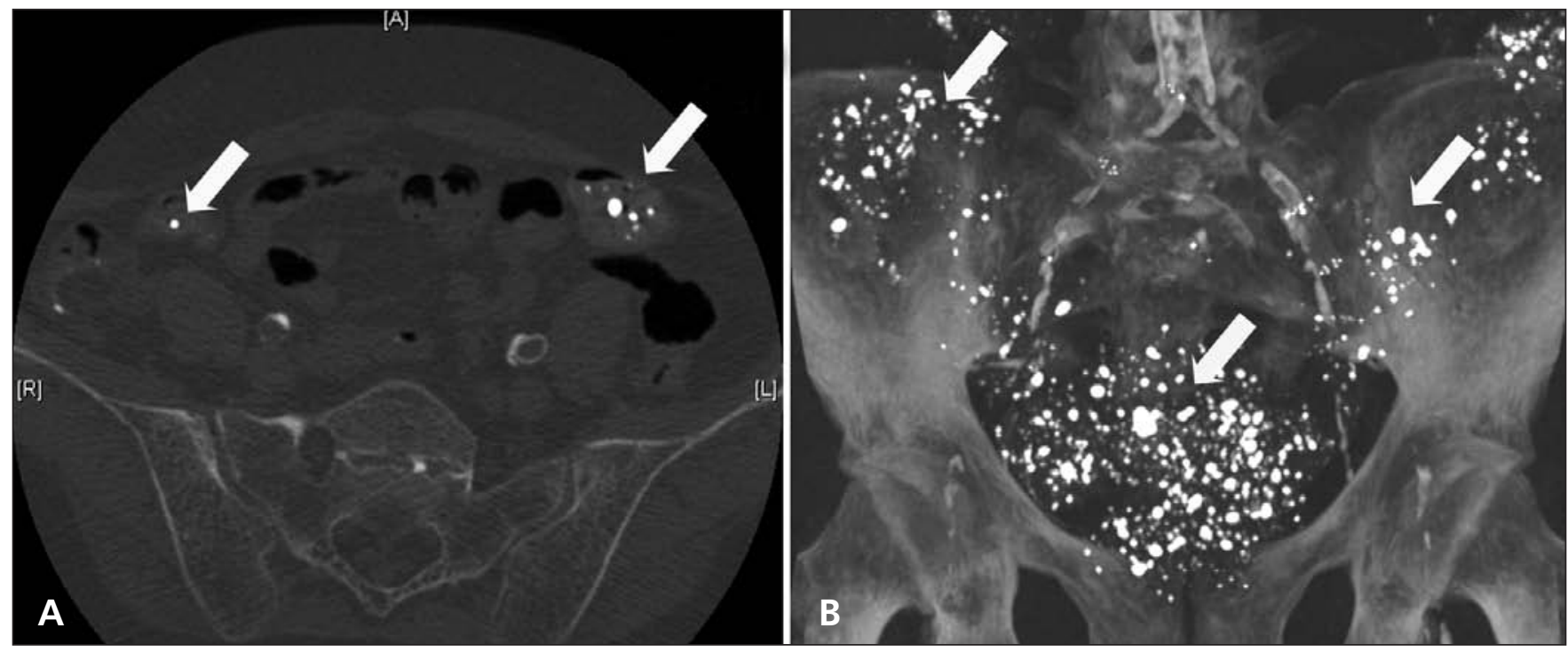

Figure 1: Computed tomographic image (A) and coronal projection reformation (B) of the abdomen and pelvis of a 54-year-old woman with chronic renal failure. Multiple intense radiopaque structures of varying size (arrows) can be seen in the small and large intestines.

$\mathrm{P}$ rogressive peripheral arterial disease developed in a 54-year-old woman who had been undergoing hemodialysis for six years for polycystic kidney disease. Upon diagnosis with peripheral aterial disease, she no longer qualified for the active waiting list for renal transplant. After successful treatment with percutaneous transluminal angioplasty, she was re-evaluated for the active waiting list. The evaluation included computed tomography of the abdomen and pelvis, which showed severe atherosclerotic calcifications in the abdominal aorta and iliac arteries. Multiple intense radiopaque structures of varying size were found in the small and large intestines (Figure 1). On further questioning, the patient reported that her doctor had changed her antiphosphate medication to lanthanum carbonate. She reported no changes to her bowel habits, such as constipation or diarrhea.

Lanthanum carbonate is a novel nonaluminum, noncal-

From the Departments of Nephrology and Hypertension (Schmitt, Haller) and Radiology (Lotz), Hannover Medical School, Hannover, Germany

CMAJ 2010. DOI:10.1503/cmaj.091935 cium phosphate binder that has been increasingly used in clinical practice. ${ }^{1}$ Oral administration of lanthanum carbonate inhibits absorption of phosphate from food by forming insoluble lanthanum-phosphate complexes in the intestinal tract. This results in a reduction of serum phosphate, which lowers the risk of cardiovascular complications caused by hyperphosphatemia in patients undergoing dialysis. ${ }^{1}$

Although lanthanum carbonate for reduction of phosphate is useful, it is a radiopaque metal that may appear on radiographs. ${ }^{2}$ To achieve a nonobscured abdominal image, treatment with lanthanum carbonate should be discontinued several days before abdominal imaging.

This article has been peer reviewed.

Competing interests: None declared.

\section{REFERENCES}

1. Curran MP, Robinson DM. Lanthanum carbonate: a review of its use in lowering serum phosphate in patients with end-stage renal disease. Drugs 2009;69:2329-49.

2. Pafcugová J, Horácková M, Hrasková M, et al. Radio-opaque appearance of lanthanum carbonate in a patient with chronic renal failure. Nephrol Dial Transplant 2008;23:1776-7. 